\title{
Multiple-Observation-Based Robust Channel and Doppler Estimation in High Mobility Applications
}

\author{
Md. Jahidur Rahman ${ }^{1}$ and Jiaxin Yang ${ }^{2}$ \\ ${ }^{1}$ Department of Electrical and Computer Engineering, The University of British Columbia, Vancouver, Canada V6T 1Z4 \\ ${ }^{2}$ Department of Electrical and Computer Engineering, McGill University, Montreal, Canada H3A OE9 \\ Correspondence should be addressed to Md. Jahidur Rahman; jrahman@ece.ubc.ca
}

Received 10 November 2012; Revised 11 April 2013; Accepted 12 April 2013

Academic Editor: Shinsuke Hara

Copyright (c) 2013 Md. J. Rahman and J. Yang. This is an open access article distributed under the Creative Commons Attribution License, which permits unrestricted use, distribution, and reproduction in any medium, provided the original work is properly cited.

\begin{abstract}
Channel estimation is a challenging task, especially in high mobility applications due to the rapid variation of the propagation environment. This paper presents a new technique that exploits past channel impulse responses (CIRs) in order to trace and compensate Doppler frequency in mobile applications, enabling robust estimation of time-varying channel. Based on the fact that channel taps at different time instants can be fitted with a sinusoidal wave, a joint estimator is proposed to estimate the channel parameters. Therefore, the efficiency of the channel estimation can be improved and stringent delay requirements for the communication systems can also be satisfied. Simulation results show that system performance in terms of bit error rate (BER) is significantly improved with the proposed algorithm.
\end{abstract}

\section{Introduction}

Channel estimation is of crucial importance for reliable coherent detection in high mobility applications, such as vehicular-to-vehicular (V2V) or vehicular-to-infrastructure (V2I) communications $[1,2]$. It is complicated due to the large amount of Doppler frequency experienced by moving vehicles, and, in turn, the rate of channel variation can be sufficiently high, leading to poor channel estimation performance. Therefore, channel estimation performance in high mobility applications is generally poor. In an effort, past observations of channel impulse responses (CIRs) can be exploited to improve the channel estimation performance. However, in highly mobile environments, the direct combination of multiple observed CIRs is not effective due to the phase rotation introduced by Doppler frequency, and, hence the combination is noncoherent. Therefore, the estimation of Doppler frequency is essential to improve the channel estimation performance in mobile communication. If the total amount of Doppler frequency or change in Doppler frequency can be estimated exploiting observed past CIRs, the channel can be tracked even after the elapse of the channel coherence time, which will make communication more robust in mobile applications.

One of the first studies reported in the literature for the estimation of Doppler frequency is [3], where the authors proposed estimation techniques using both complex and real envelop of the received signal. Another method to estimate the Doppler frequency in the presence of speckle and receiver noise was presented in [4]. This estimation technique is based on the correlation of the signal power spectra with an arbitrary weighting function and specifically tailored for Synthetic Aperture Radar (SAR) data. On the other hand, an estimation technique for OFDM-based transmission that relied on finding the autocorrelation function of time domain channel estimates was studied in [5]. Besides, a good review of Doppler estimation techniques can be found in [6]. However, most of these techniques are based on obtaining the autocorrelation function (ACF) of the received signals or channel estimates. Once the ACF is estimated, different methods can be used to calculate the Doppler spread as discussed in [6]. However, it requires precise estimation of 


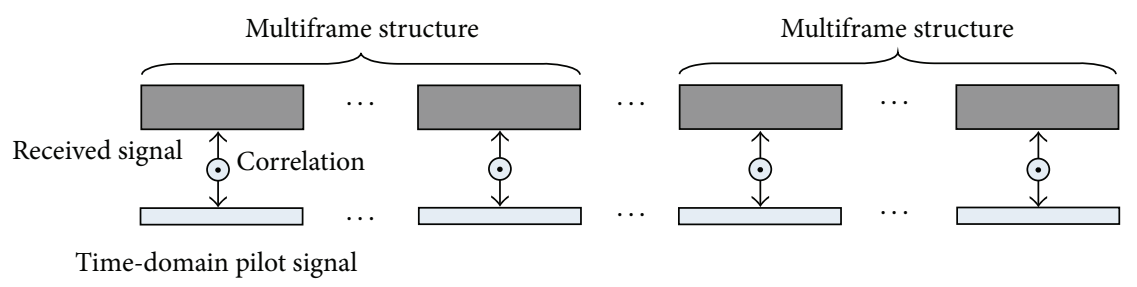

FIGURE 1: Multiframe based observation of channel impulse responses.

the time lag which often becomes erroneous due to the presence of line-of-sight component, nonisotropic scatterers, and so forth [6].

In this paper, we have studied a joint CIR and Doppler frequency estimator that exploit multiple observations of the past CIRs, which can be used to track the CIR in the current estimation period. At first, a multiframe time-domain correlation technique is used to obtain the time-domain estimates of the past CIRs, as shown in Figure 1. Thereafter, these observations of immediate past CIRs are exploited to model the variation of the Doppler frequency in the current estimation period, as described in Section 3. Comparing it to the sequential estimation, a joint estimator is more efficient as it takes into account the relation between the parameters and reduces the overhead necessary for achieving a certain system performance [7]. This is especially important in burst transmission systems, where the data burst is relatively short and the overhead of the preamble and pilot data must be kept to a minimum as in vehicular communications. Because of the joint estimation of the channel parameters, this technique can also satisfy the delay requirements for vehicular communications [8]. In our analysis, we have considered that multipath components in a channel profile may experience different Doppler shifts and may or may not vary from one observation instant to another. Therefore, the research problem is to estimate the CIR and Doppler frequency jointly, based on multiple observations of past CIRs. It should be noted that time-domain implementation improves the estimator performance because of the reduced number of channel parameters to be estimated and the exact modeling of the phase rotation suffered by the Doppler frequency [9]. Therefore, the data reception performance can be improved by estimating the variation of the channel model in a mobile environment.

Note that the proposed method does not rely on the estimation of ACF as in conventional techniques, that is, prone to erroneous estimation of Doppler due to model imperfections such as presence of line-of-sight component and nonisotropic scattering. In addition, the proposed method uses local timedomain pilot signals to obtain multiframe observations of the channel, therefore free of model imperfections, that is, a major drawback for the conventional techniques [6]. If we compare the computation complexity of the proposed algorithm against the conventional schemes, the proposed algorithm is much simpler to implement. In conventional algorithm, the estimated channel needs to be correlated, that is, obtaining the ACF in order to determine the time lag that is necessary to estimate the Doppler frequency. In the proposed algorithm, on the other hand, we can simply use the channel parameters observed from past CIRs in order to determine the Doppler for the current estimation period. Therefore, the proposed algorithm provides an accurate estimation of Doppler frequency as seen from our simulation results with much lower computation complexity than the conventional schemes.

The rest of the paper is organized as follows. Section 2 describes the multiframe-based channel observation technique. The estimation of channel parameters is described in Section 3. The initializations of the parameters are described in Section 4. The channel estimation technique with varying Doppler for the past observations is discussed in Section 5. Simulation results for this study are presented in Section 6. Finally, the paper is summarized in Section 7.

\section{Multiframe Channel Observation}

Let us consider a multipath channel as shown in Figure 2. Each path is affected by a different Doppler frequency at different time instant and can be modeled as follows [10].

$$
h(t)=\sum_{l=0}^{L-1} a_{t, l} e^{j\left(2 \pi \zeta_{t, l} t+\gamma_{t, l}\right)},
$$

where $a_{t, l}, \gamma_{t, l}$, and $\zeta_{t, l}$ denote the time-dependent path amplitude, initial phase, and Doppler frequency for the $l$ th tap at time instant $t$, and $L$ is the total number of paths for any given CIR.

Over a short interval of time, for example, when observation times $t_{1}$ and $t_{2}$ are very close, we can assume that the magnitudes of the multipath components do not vary significantly. Considering that the multipath amplitudes do not vary significantly over a short observation of the channel, it can be written that $\Gamma\left(a_{t_{1}, l}\right) \approx \Gamma\left(a_{t_{2}, l}\right)$, where $\Gamma$ is the magnitude operation and $a_{t_{1}, l}$ and $a_{t_{2}, l}$ are the amplitudes of the multipath components at time instants $t_{1}$ and $t_{2}$. In first part of our analysis, we have considered that the Doppler frequency and initial phase do not change over the observation period.

A multiframe technique is used to obtain multiple observations of the past CIRs [11]. This technique is useful to obtain multiple observations of the channel at the same time and exploit these observations to estimate Doppler frequency and channel in the current estimation period.

In order to obtain the reference CIR, we have used time-domain channel estimation for the OFDM system, 


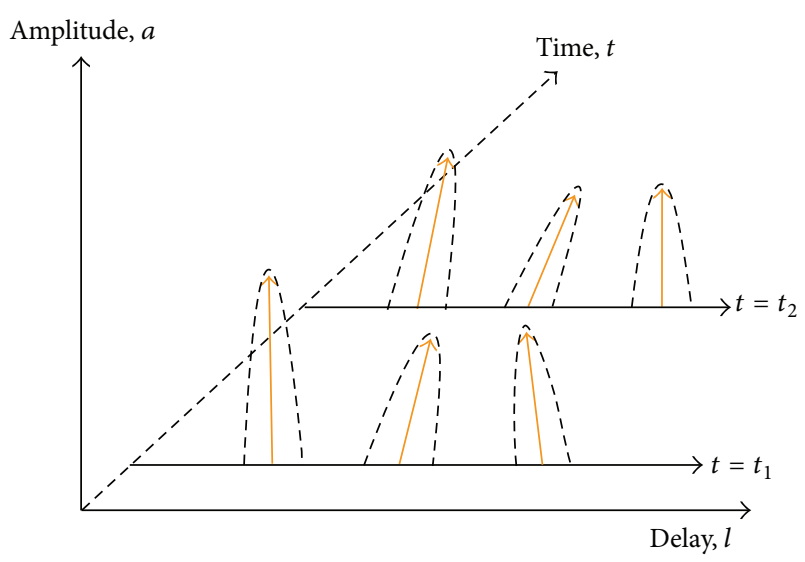

FIgURE 2: Typical multipath channel impulse responses observed at different time instants.

tailored for DVB-T system [12]. Let us consider that the time domain data and pilot signals are denoted by $d(n)$ and $p(n)$, respectively. The pilot tones are multiplexed with the data signal in the frequency domain and assume that combined signal in time-domain is given by $x(n)$. Therefore, the received time-domain signal at successive time instants, $y(t, n)$, follows

$$
y(t, n)=x(n) \otimes h(t, n)+w(t, n),
$$

where $\otimes$ denotes the convolution operation and $w$ is the discrete AWGN noise. The time-domain pilot signal can be used to correlate with the received time-domain signal in order to obtain an estimation of the channel. Considering channel taps $l=1,2, \ldots, L$, the time-domain correlation output between local pilot and $m$ th received frame is given by

$$
\begin{aligned}
\widehat{h}\left(t_{m} ; l\right)= & \sum_{n=1}^{N} y\left(t_{m}, m N+n\right) \odot p\{m N+n\} \\
= & \sum_{n=1}^{N}\left[x(m N+l) \otimes h\left(t_{m}, l\right)\right. \\
& \left.\quad+w_{m}\left(t_{m}, m N+l+n\right)\right] \odot p\{m N+n\} \\
= & h\left(t_{m} ; l\right)+\widehat{w}\left(t_{m} ; l\right),
\end{aligned}
$$

where $\odot$ denotes the correlation operation, $\widehat{h}\left(t_{m} ; l\right)$ denotes the estimated time-domain channel in the $m$ th frame and $l$ denotes each path in the CIR, and $\widehat{w}\left(t_{m} ; l\right)$ denotes the correlation of the pilot signal with the inherent noise component. Note that the definition of frame depends on how frequent channel needs to be observed. The estimated channel $\widehat{h}\left(t_{1}, t_{2}, \ldots, t_{M} ; l\right) \equiv h_{1}, h_{2}, \ldots, h_{M}$ at successive time instants, $t_{1}, t_{2}, \ldots, t_{M}$, will be used in the next section to estimate the channel parameters in the current estimation period.

\section{Estimation of Channel Parameters}

Let us consider that the channel samples observed at time instants $t_{1}, \ldots, t_{M}$ are given by $h_{1}, \ldots, h_{M}$. Considering a single path at different observation instants, the path amplitude, Doppler frequency, and initial phase can be modeled as

$$
\begin{aligned}
h_{m}[a, \zeta, \gamma] & =a \cos \left\{2 \pi \zeta t_{m}+\gamma\right\} \\
& =A_{1} \cos 2 \pi \zeta t_{m}+A_{2} \sin 2 \pi \zeta t_{m} \\
& =h_{m}[\theta],
\end{aligned}
$$

where $A_{1}$ and $A_{2}$ are unknown constants, $A_{1}=a \sin \gamma$ and $A_{2}=a \cos \gamma$, and $\theta$ is the set of the channel parameters. The channel observation instant and path numbers are dropped for simplification. For the estimation of channel parameters, here we have followed IEEE Standard 1057 [13]. Note that here in this section we have considered only the real part of the complex channel. Similar analysis holds for imaginary part of the channel estimate as well.

Let us assume that an estimate of Doppler frequency in iteration $i$, say $\widehat{\zeta}_{i}$, of $\zeta$ is available. Therefore, a Taylor series expansion around the estimate $\widehat{\zeta}_{i}$ gives [14]

$$
\cos t_{m}=\cos 2 \pi \widehat{\zeta}_{i} t_{m}-t_{m} \sin \hat{2} \pi \zeta_{i} t_{m} \Delta \zeta_{i}
$$

Similarly,

$$
\sin t_{m}=\sin 2 \pi \widehat{\zeta}_{i} t_{m}+t_{m} \cos 2 \pi \widehat{\zeta}_{i} t_{m} \Delta \zeta_{i},
$$

where $\Delta \zeta_{i}=\zeta_{i}-\widehat{\zeta}_{i}$

Using the above two approximations in (4), we get that

$$
\begin{aligned}
h_{m}\left[\theta_{i}\right] \approx & A_{1} \cos 2 \pi \widehat{\zeta}_{i} t_{m}+A_{2} \sin 2 \pi \widehat{\zeta}_{i} t_{m} \\
& -A_{1} t_{m} \Delta \zeta_{i} \sin 2 \pi \widehat{\zeta}_{i} t_{m}+A_{2} t_{m} \Delta \zeta_{i} \cos 2 \pi \widehat{\zeta}_{i} t_{m},
\end{aligned}
$$

where $\theta_{i}$ is the parameter vector.

The above equation is nonlinear in the parameters, but it may be linearized using the assumption that Doppler is constant over the observation period; that is, $\Delta \zeta_{i}=\zeta-\widehat{\zeta} \approx$ 0 . Putting available estimates of $A_{1}$ and $A_{2}$ from previous iteration, that is, $A_{1, i-1}$ and $A_{2, i-1}$, the above equation results in an equation linear in the components of $\theta_{i}$. Therefore, observing the past observations, the following can be written:

$$
h=\widehat{Q}_{i} \theta_{i}
$$

where $\widehat{Q}_{i}$ is the $M \times 3$ matrix, and denoting $2 \pi \widehat{\zeta}_{i} t_{i}=\widehat{\Omega}_{i}, \widehat{Q}_{i}$ can be written as

$$
\left[\begin{array}{ccc}
\cos \widehat{\Omega}_{1} & \sin \widehat{\Omega}_{1} & -\widehat{A}_{1, i-1} t_{1} \sin \widehat{\Omega}_{1}+A_{2, i-1} t_{1} \cos \widehat{\Omega}_{1} \\
\cos \widehat{\Omega}_{2} & \sin \widehat{\Omega}_{2} & -\widehat{A}_{1, i-1} t_{2} \sin \widehat{\Omega}_{2}+A_{2, i-1} t_{2} \cos \widehat{\Omega}_{2} \\
\vdots & \vdots & \vdots \\
\cos \widehat{\Omega}_{M} & \sin \widehat{\Omega}_{M} & -\widehat{A}_{1, i-1} t_{M} \sin \widehat{\Omega}_{M}+A_{2, i-1} t_{M} \cos \widehat{\Omega}_{M}
\end{array}\right] .
$$

It is obvious that in order to obtain a unique solution for each of the parameters, at least three observations are 
necessary. The simple technique is to repeatedly solve the above linear system; that is, at iteration $i, \widehat{Q}_{i}$ is used to obtain a new set of estimates $\widehat{\theta}_{i}$ and the solution is given by

$$
\theta_{i}=\left(\widehat{Q}_{i}^{T} \widehat{Q}_{i}\right)^{-1} \widehat{Q}_{i}^{T} h
$$

The algorithm follows an iterative process to find the parameters that minimize the sum of squared differences as the following:

$$
\begin{aligned}
& e=\sum_{m=1}^{M}\left(h_{m}-h_{m}[\theta]\right)^{2} \\
& \text { S.T. } e<e_{\mathrm{thr}},
\end{aligned}
$$

where $e_{\text {thr }}$ is the estimation error threshold set to achieve a certain estimation performance.

Once $A_{1}$ and $A_{2}$ are known, initial phase $(\gamma)$ and the amplitude $(a)$ from the $i$ th iteration can be obtained as follows:

$$
\begin{aligned}
& \gamma_{i}=\tan ^{-1} \frac{A_{1, i}}{A_{2, i}}, \\
& a_{i}=\frac{A_{1, i}}{\sin \gamma_{i}}=\frac{A_{2, i}}{\cos \gamma_{i}} .
\end{aligned}
$$

\section{Initializations of the Parameters}

4.1. Initial Guess for the Amplitude, a. The initial guess for $a$ in the minimization problem plays an important role in the convergence of the algorithm. In order to have a faster convergence, the initial guess can be obtained by averaging the amplitudes of the past CIRs. Due to the averaging, the impact of noise will be reduced as well [15]. The initial guess for channel magnitude, $a_{i}$, can be derived as

$$
\tilde{a}_{i}(t, l)=\frac{1}{M} \sum_{m=1}^{M}\left\{a_{m}(t, l)\right\},
$$

where $M$ indicates the number of CIRs considered from the past observations.

4.2. Initial Guess for the Doppler Frequency, $\zeta$. For a moving vehicle, there could be a constrained set on the Doppler frequency based on the mobility pattern of the vehicle. Basically, it could be determined by the maximum acceleration or deceleration of the vehicle. Therefore, the Doppler shift can be initialized based on the study of mobility pattern in a particular scenario, that is, highway. The constrained can be formulated as

$$
\begin{gathered}
\zeta-\Delta \zeta_{\max }<\zeta_{i}<\zeta+\Delta \zeta_{\max }, \\
\zeta-\frac{\cos \gamma_{l}}{\lambda}\left\{\frac{-\Delta v(t)}{\Delta t}\right\}<\zeta_{i}<\zeta+\frac{\cos \gamma_{l}}{\lambda}\left\{\frac{\Delta v(t)}{\Delta t}\right\},
\end{gathered}
$$

where $\Delta \zeta_{\max }$ is the maximum change in the Doppler shift because of the change in velocity or angle of arrival. The initial value of the Doppler frequency can be chosen within this range for faster and more accurate convergence.
4.3. Initial Guess for the Initial Phase, $\gamma$. If the Doppler phase remains constant over the observation period, taking the difference between the phase of the successive channel estimate would leave the initial phase. For a faster convergence, guess for initial phase can be computed from the differential phase rotations obtained from the past CIRs as follows:

$$
\bar{\gamma}_{1}=\left[\bar{\phi}_{1}-\bar{\phi}_{2}\right]^{T}
$$

where $\phi$ is the combined phase shift. It includes the Dopplerinduced phase shift and initial phase and is given by $\phi_{t_{1}, l}=$ $2 \pi \zeta_{t_{1}, l} t_{1}$. Similarly, for the next two observations, we can write

$$
\bar{\gamma}_{2}=\left[\bar{\phi}_{2}-\bar{\phi}_{3}\right]^{T}
$$

In general, these successive differences for $M$-observed channels can be averaged to obtain a guess of the initial phase for the minimization problem as follows:

$$
\tilde{\gamma}_{i}=\frac{1}{M-1} \sum_{m=1}^{M-1} \bar{\gamma}_{m}
$$

It should be noted that the convergence would be much faster than the conventional joint estimation technique, as in the conventional technique only one estimation is used to obtain the initial guesses of the channel parameters.

4.4. Determination of Number of Required Observations, $M$. The performance of channel estimation following this technique will be determined by the number of observations obtained as well as the estimation error introduced in the past observations. Let us consider that past observed CIR has an error $e$, which is a zero-mean Gaussian noise with variance $\sigma^{2}$. Therefore, according to IEEE STD 1057, the lower bound of the performance for this estimator can be found by CramerRao bound (CRB) as follows:

$$
\operatorname{CRB}(\widehat{\zeta})=\left(\frac{n}{2 \pi t_{n}}\right)^{2} \frac{12}{\operatorname{SNR}\left(M^{2}-1\right) M}
$$

where SNR $=\left(A_{1}^{2}+A_{2}^{2}\right) / 2 \sigma^{2}, n$ is the observation instant, $t_{n}$ is the observation interval, and $M$ is the total number of observations. It is seen that the CRB is inversely proportional to the total number of observations.

It is important that we study the number of observations needed to obtain certain performance threshold of the estimator. For a certain $\mathrm{CRB}$, the above equation can be rewritten as

$$
M^{3}-M-C=0,
$$


where $C=\left(n / 2 \pi t_{n}\right)^{2}(12 / \mathrm{SNR} \times \operatorname{CRB}(\widehat{\zeta}))$. Solving this equation analytically, we get the following three solutions for the number of the observations, $M$,

$$
M=\left\{\begin{array}{l}
\frac{1}{6} S+\frac{2}{S}, \\
-\frac{1}{12} S-\frac{3}{S}+\frac{\sqrt{-j}}{12} S, \\
-\frac{1}{12} S-\frac{3}{S}-\frac{\sqrt{-j}}{12} S,
\end{array}\right.
$$

where $S=\left[108 \times C+12 \times \sqrt{\left(-12+81 C^{2}\right)}\right]^{1 / 3}$. As the number of the observations will be a real positive number and assuming the condition $81 C^{2}-12 \geq 0$, we have the following three real solutions:

$$
M=\left\{\begin{array}{l}
\frac{S}{6}+\frac{2}{S}, \\
-\frac{S}{12}-\frac{3}{S}, \\
-\frac{S}{12}-\frac{3}{S} .
\end{array}\right.
$$

The above solutions provide the bounds on the number of the observations required in order to maintain the CRB for a given SNR level and sampling frequency.

\section{Estimator Performance with Varying Doppler}

In this section, we will study the change in Doppler frequency over the observation period and its impact on the performance of the estimator. If the Doppler frequency does not change appreciably over the observation period, the previous technique can be used to estimate the channel parameters. However, the Doppler frequency may change over the observation period, depending on the mobility pattern of the users and angle of arrivals for each path.

It is obvious that the speed of a vehicle is a time-varying function and so is the Doppler frequency for each path. The relationship between the change of Doppler frequency and the speed of a vehicle can be derived as follows [16]:

$$
\frac{d \zeta_{t, l}}{d t}=\frac{\cos \gamma_{t, l}}{\lambda} \frac{d v(t)}{d t}+\frac{v(t)}{\lambda} \frac{d}{d t}\left\{\cos \gamma_{t, l}\right\}
$$

where $\Delta t=t_{2}-t_{1}$ is the time difference between two successive channel sampling instants. Therefore, it can be seen that change in Doppler frequency depends on the change in the speed or change in the angle of arrival. Even if there is no change in speed within this short-time period $\Delta t$, it is possible that multipath will have different angles of arrivals. However, with no significant change in speed within the short interval of time $\Delta t$, the above equation can be written as

$$
\delta \zeta_{t_{m}, l}=\frac{v(t)}{\lambda} \frac{d}{d t}\left\{\cos \gamma_{t, l}\right\} .
$$

The above equation would determine the variation of Doppler frequency for each multipath in a CIR over the time difference between successive CIRs. Under high mobility application, in fact, a short interval of time may introduce a significant variation of Doppler shifts for the successive CIRs depending on the duration of the data frame structure; however, change in Doppler frequency will be dominated by the change in the angle of arrivals for each multipath.

Therefore, considering the variation in Doppler, (4) can be written as

$$
\begin{aligned}
h_{m}[a, \zeta, \gamma] & =a \cos \left\{2 \pi \zeta_{t_{m}, l}^{\prime} t_{m}+\gamma_{t_{m}, l}\right\} \\
& =A_{1} \cos 2 \pi \zeta_{t_{m}, l}^{\prime} t_{m}+A_{2} \sin 2 \pi \zeta_{t_{m}, l}^{\prime} t_{m} \\
& =h_{m}\left[\theta^{\prime}\right],
\end{aligned}
$$

where $\zeta_{t_{m}, l}^{\prime}=\zeta \pm \delta \zeta_{t_{m}, l}=\rho \zeta$. Here, $\rho$ denotes the correlation in Doppler frequency over the past observation instants and $\delta \zeta$ is the amount of variation. When $\rho=1$, it denotes the constant Doppler case. In order for this problem to be linearized, the following condition has to be satisfied:

$$
\zeta_{m}-\widehat{\zeta}_{m}=\Delta \zeta_{i} \approx 0
$$

where $\zeta_{m}$ and $\widehat{\zeta}_{m}$ denote the original and estimated Doppler frequencies. It also means that $\rho \approx 1$. Once these conditions are satisfied, a linear equation similar to (7) is formulated and can be solved to obtain the channel parameters. However, if all of the above conditions are not satisfied, the estimation of channel parameters remains a nonlinear problem. Therefore, the estimated parameter will have an error, which can be modeled as follows:

$$
h_{m}^{\prime}[\theta]=h_{m}[\theta]+e^{\prime},
$$

where $h_{m}^{\prime}[\theta]$ denotes the estimated set of parameters when the Doppler frequency is slightly different over the past observations and $e^{\prime}$ is corresponding error when compared to the case of no Doppler variation. In this case, this error can be amalgamated with the error threshold $\left(e_{\text {thr }}\right)$ in the minimization process. The new threshold can be written as

$$
e_{\mathrm{thr}}^{\prime}=e_{\mathrm{thr}}+e^{\prime} .
$$

The small variation in Doppler shift over the observation period can be considered as a noise in the estimation process. Therefore, the noise introduced in the estimation process will be higher than the stable Doppler case. Let us consider that the variance of the noise in this case is given by $\sigma^{\prime 2}=\sigma^{2}+\Delta \sigma^{2}$, where $\Delta \sigma^{2}$ is the noise introduced in the past observation of the channel due to varying Doppler and can be considered as zero-mean Gaussian variable when there are sufficient numbers of observations. Therefore, CRB for this estimator under varying Doppler can be found as follows:

$$
\operatorname{CRB}(\widehat{\zeta})=\left(\frac{n}{2 \pi t_{n}}\right)^{2} \frac{12}{\operatorname{SNR}^{\prime}\left(M^{2}-1\right) M},
$$

where $\mathrm{SNR}^{\prime}=\left(A_{1}^{2}+A_{2}^{2}\right) / 2 \sigma^{\prime 2}$. 


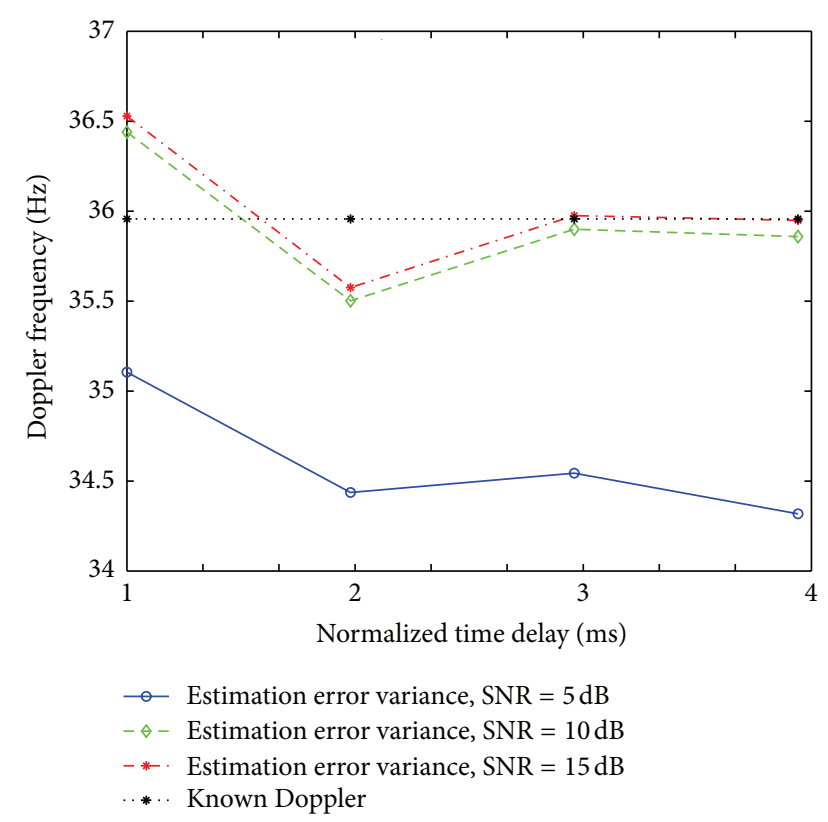

Figure 3: Comparison of estimated Doppler frequency with true Doppler in presence of different estimation errors (constant Doppler case).

\section{Simulation Results}

Digital Video Broadcasting-Terrestrial (DVB-T) system is used to evaluate the performance of the proposed technique. It is assumed in the simulation that the past channel responses may experience a constant or varying Doppler under a receiver velocity from $50 \mathrm{~km} / \mathrm{h}$ to $100 \mathrm{~km} / \mathrm{h}$. DVB$\mathrm{T}$ standard transmission for $2 \mathrm{~K}$ mode with $8 \mathrm{MHz}$ bandwidth and $800 \mathrm{MHz}$ carrier frequency is used for the simulation of the proposed technique. The sampling period for the chosen data transmission is $7 / 64 \mu \mathrm{s}$. In this paper, we have considered an arbitrary statistical channel as well as widely known International Telecommunication Union (ITU) Vehicular Channel Model B to evaluate the system performance [17]. It is assumed that the arbitrary channel is normalized and follows uniform power delay profile.

Figure 3 shows the comparison of estimated and known Doppler frequency with estimation error introduced by the past observations of the CIRs. In this channel model, we assumed that each path experiences same Doppler frequency. It is important to note that as the estimation error variance decreases, the estimated Doppler frequency approaches the true Doppler frequency.

Figure 4 shows the comparison of estimated and known Doppler frequency with estimation error introduced by the past observed CIRs with the Doppler frequency set to be different for different channel taps. Similar to the Figure 3, as the estimation error variance decreases, the estimated Doppler frequency approaches the true Doppler frequency. Therefore, it is expected that the system performance will improve after compensating the Doppler impairment as shown in the next few simulations.

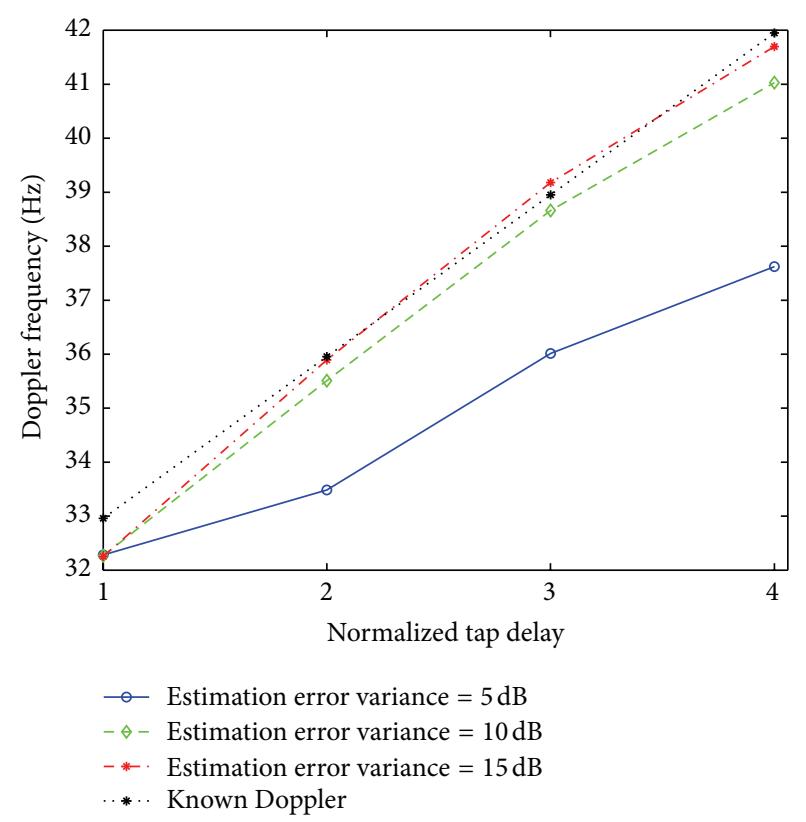

Figure 4: Comparison of estimated Doppler frequency with true Doppler in presence of different estimation errors (varying Doppler case).

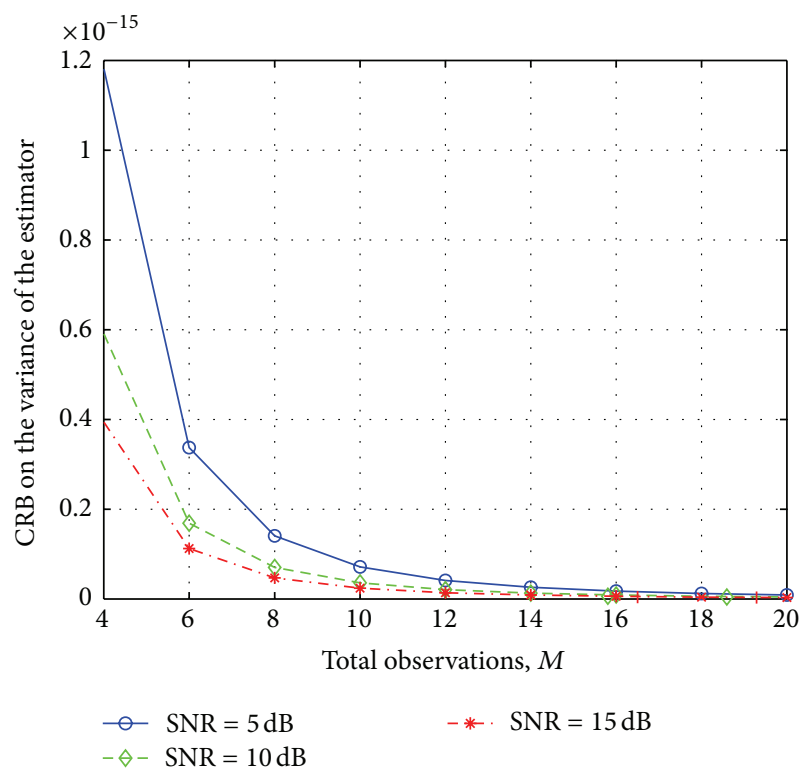

Figure 5: Cramer-Rao bound on the variance of the proposed channel estimator.

Figure 5 shows the CRB of the estimator versus the number of past observations under different SNR. It is seen that with an increase in the number of past observations, the CRB decreases. In addition, as the SNR increases, the CRB also decreases, indicating the improvement of the estimation performance.

Figure 6 presents the performance of the DVB-T system transmitting in $2 \mathrm{k}$ mode at a carrier frequency of $800 \mathrm{MHz}$. We have first adopted the widely known ITU Vehicular 


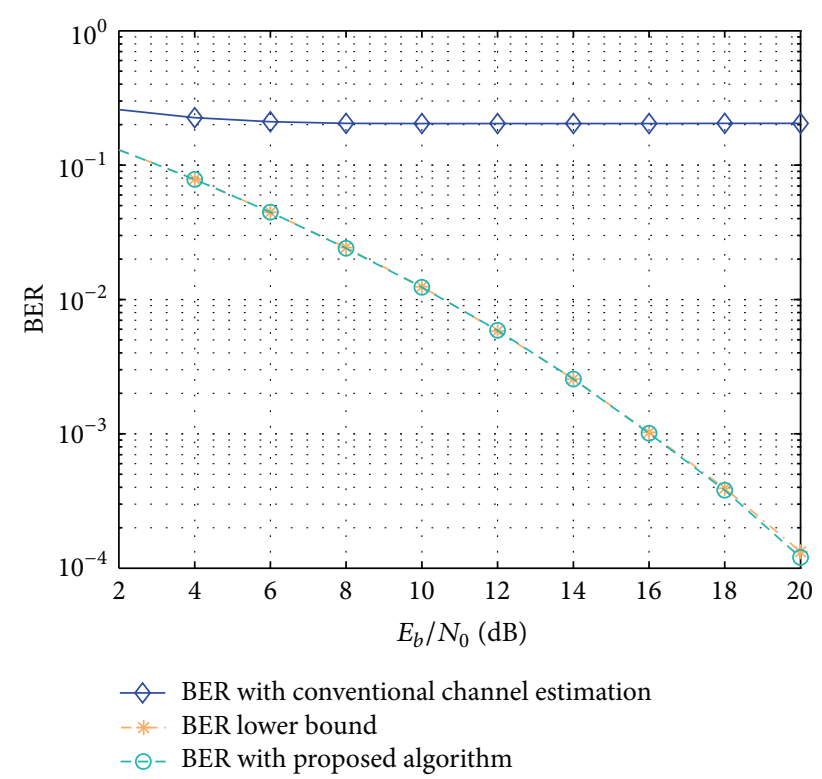

FIGURE 6: Improvement of DVB-T system performance in terms of BER with the proposed algorithm in ITU Vehicular Channel Model B.

Channel Model B. It is seen that the BER performance is significantly improved once the Doppler impairment is compensated on the estimated channel. The conventional channel estimation shows a large BER due to the nature of the ITU channel model. In conventional scheme, Doppler induced impairment is not compensated after the channel estimation, and therefore the signal constellation is rotated which caused error on the data demodulation. On the other hand, we have used the fact that estimated Doppler can be used to compensate the impairment caused by Doppler frequency itself which eventually contributes to the improvement of BER. As seen from this result, the BER performance with the proposed algorithm is very close to the lower bound (Doppler impairment can be perfectly compensated), indicating the robust performance of the proposed Doppler estimation algorithm.

Figure 7 presents the performance of the DVB-T system under the same system parameters as above, transmitting in $2 \mathrm{k}$ mode at $800 \mathrm{MHz}$. However, this time we have simulated a worse channel with 200 taps following normalized uniform power delay profile. It is seen that the proposed algorithm performs better even under this worse channel condition. Similarly, as shown in the above figure, the BER performance with the proposed algorithm is also very close to the lower bound, indicating the robust performance of the proposed algorithm. The performance improvement is about $4 \mathrm{~dB}$ in the low SNR region and that is more than $6 \mathrm{~dB}$ in the high SNR region.

\section{Conclusions}

In this paper, a robust Doppler frequency and channel estimation technique is presented that is, suitable for mobile communications. The technique exploits past observed CIRs

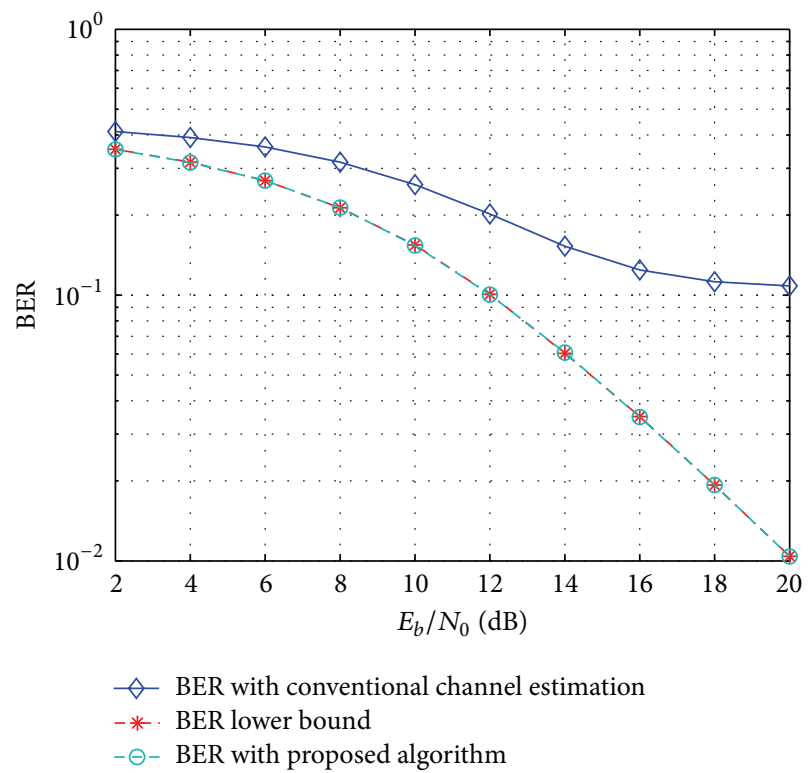

FIGURE 7: Improvement of DVB-T system performance in terms of BER with the proposed algorithm for an arbitrary channel, under a receiver mobility of $100 \mathrm{~km} / \mathrm{h}$.

in order to jointly estimate channel parameters. It is found that the estimated Doppler frequency is very close to the known Doppler frequency as observed in the simulation results. Further, the Doppler frequency estimation algorithm has been evaluated with estimation error variance introduced by the past CIRs. As expected, it is found that with the decrease of estimation error, the Doppler frequency estimation becomes more accurate. $\mathrm{CRB}$ of the proposed channel estimator is also evaluated with the number of past observations under different SNR values. It is observed that even in highly mobile channels, with the compensation of Doppler frequency-induced impairment, the system BER performance can be significantly improved. In addition, because of the joint estimation of the channel parameters, the estimation is more efficient and much simpler to implement. The proposed technique is suitable for emerging V2V or V2I communication systems, where delay requirement and high reliability are essential.

\section{Acknowledgment}

This work was supported in part by a Canada Graduate Scholarship-Doctoral (CGSD) from the Natural Sciences and Engineering Research Council (NSERC), Canada.

\section{References}

[1] N. Goertz and J. Gonter, "Limits on information transmission in vehicle-to-vehicle communication," in Proceedings of the IEEE Vehicular Technology Conference (VTC '11), pp. 1-5, Spring, 2011.

[2] H. Trivedi, P. Veeraraghavan, S. Loke, H. P. Le, and J. Singh, "A survey of lower technologies for vehicle-to-vehicle communication," in Proceedings of the IEEE Malaysia International Conference on Communicaitons, pp. 441-446, 2009. 
[3] P. A. Bello, "Some techniques for the instantaneous realtime measurement of multipath and Doppler spread," IEEE Transactions on Communication Technology, vol. 3, no. 3, pp. 285-292, 1965.

[4] R. Bamler, "Doppler frequency estimation and the Cramer-Rao bound," IEEE Transactions on Geoscinece and Remote Sensing, vol. 29, no. 3, pp. 385-390, 1991.

[5] T. Yücek, R. M. A. Tannious, and H. Arslan, "Doppler spread estimation for wireless OFDM systems," in Proceedings of the IEEE/Sarnoff Symposium on Advances in Wired and Wireless Communication, pp. 233-236, April 2005.

[6] C. Tepedelenlioğlu, A. Abdi, G. B. Giannakis, and M. Kaveh, "Estimation of Doppler spread and signal strength in mobile communications with applications to handoff and adaptive transmission," Wireless Communications and Mobile Computing, vol. 1, no. 2, pp. 221-242, 2001.

[7] H. Nguyen, T. Le-Ngoc, and C. C. Ko, "RLS-based joint estimation and tracking of channel response, sampling, and carrier frequency offsets for OFDM," IEEE Transactions on Broadcasting, vol. 55, no. 2, pp. 84-94, 2009.

[8] X. Zhang, F. Xie, W. Wang, and M. Chatterjee, "TCP throughput for vehicle-to-vehicle communications," in Proceedings of the IEEE International Conference on Communications and Netwroking (ChinaCom '06), pp. 1-5, 2006.

[9] M. M. Freda, J. F. Weng, and T. Le-Ngoc, "Joint channel estimation and synchronization for OFDM systems," in Proceedings of the IEEE Vehicular Technology Conference (VTC '04), pp. 16731677, 2004.

[10] C. Xiao, Y. R. Zheng, N. C. Beaulieu et al., "Second-order statistical properties of the WSS Jakes' fading channe simulator," IEEE Transactions on Communications, vol. 50, no. 6, pp. 888891, 2002.

[11] M. J. Rahman, X. Wang, S. I. Park, and H. M. Kim, "Robust synchronization technique for mobile DTV broadcasting system," in Proceedings of the IEEE Conference on Computer and Information Technology (ICCIT '10), pp. 216-220, 2010.

[12] H. Minn and V. K. Bhargava, "An investigation into timedomain approach for OFDM channel estimation," IEEE Transactions on Broadcasting, vol. 46, no. 4, pp. 240-248, 2000.

[13] P. Handel, "Properties of the IEEE-STD-1057 four-parameter sine wave fit algorithm," IEEE Transactions on Instrumentation and Measurement, vol. 49, no. 6, pp. 1189-1193, 2000.

[14] E. Kreyszig, H. Kreyszig, and E. J. Norminton, Advanced Engineering Mathematics, John Wiley \& Sons, 2010.

[15] C. W. Therrien and M. Tummala, Probability for Elctrical and Computer Engineers, CRC Press, 2004.

[16] S. Haykin, Communication Sytems, John Wiley \& Sons, 2001.

[17] International Telecommunication Union-Recommendations (ITU-R) M.1225: Guidelines for evaluation of radio transmission technologies for IMT-2000. 

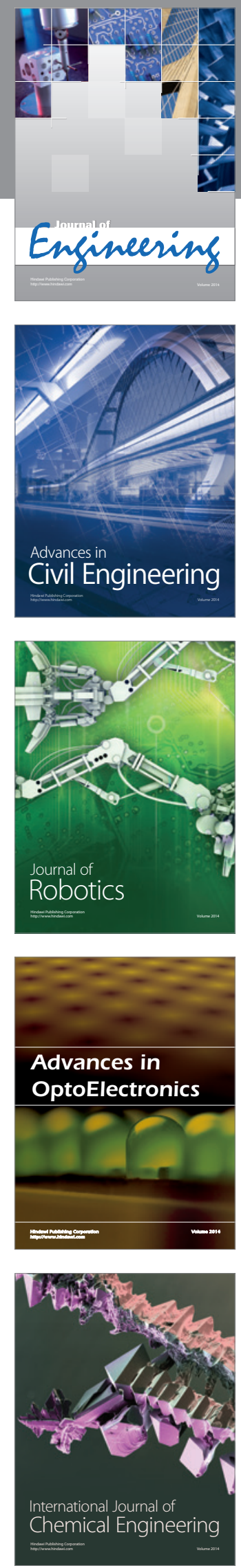

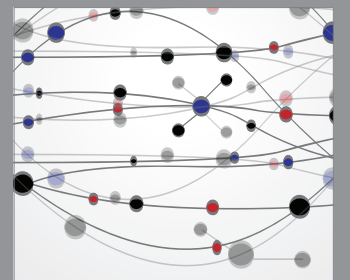

The Scientific World Journal
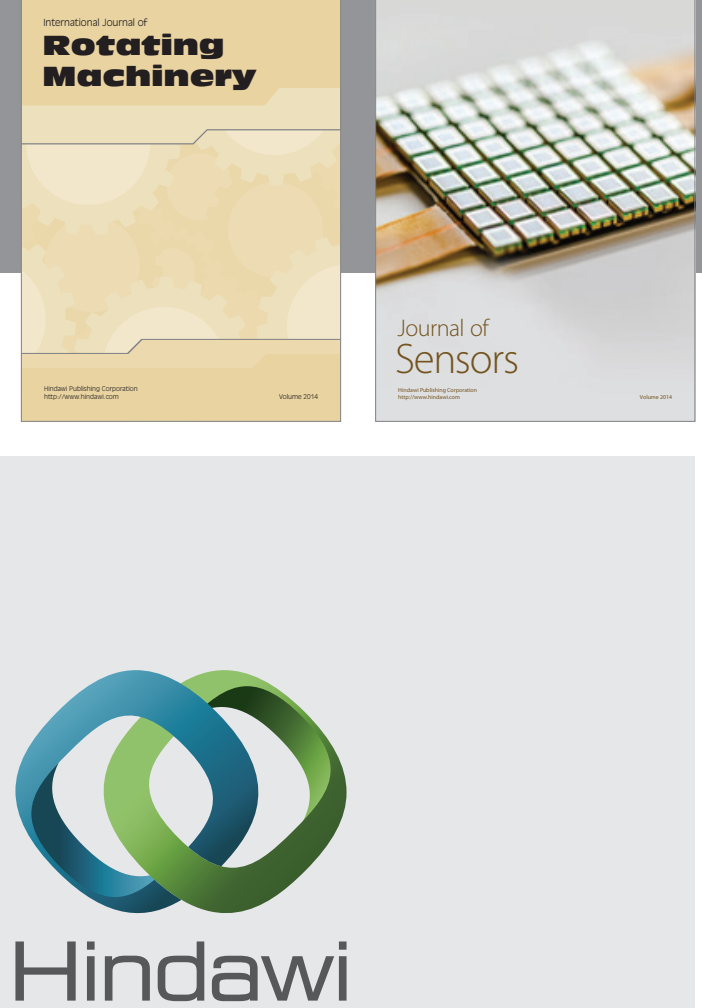

Submit your manuscripts at http://www.hindawi.com
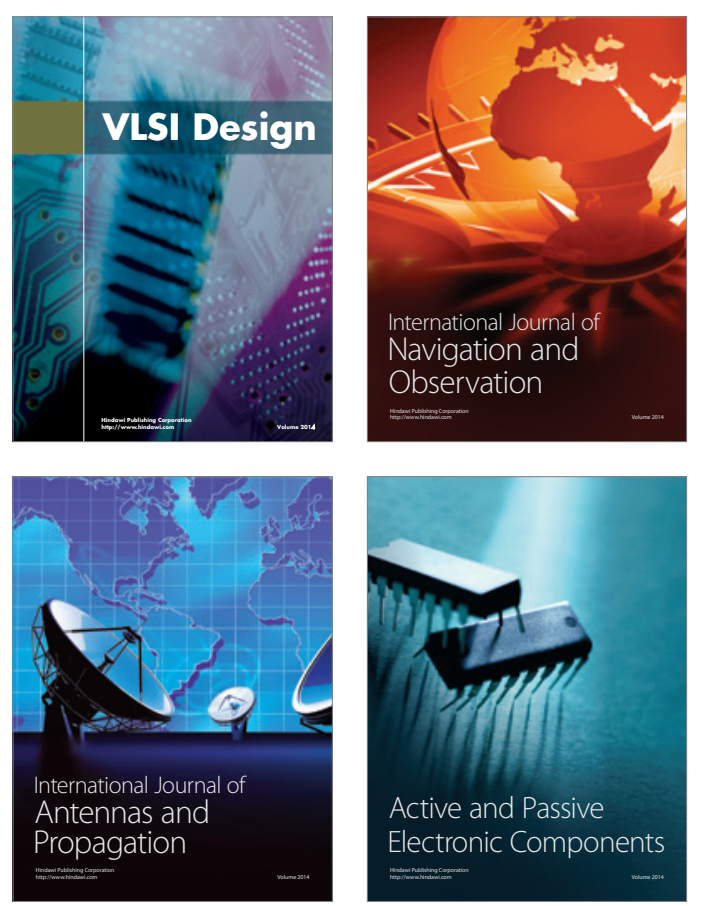
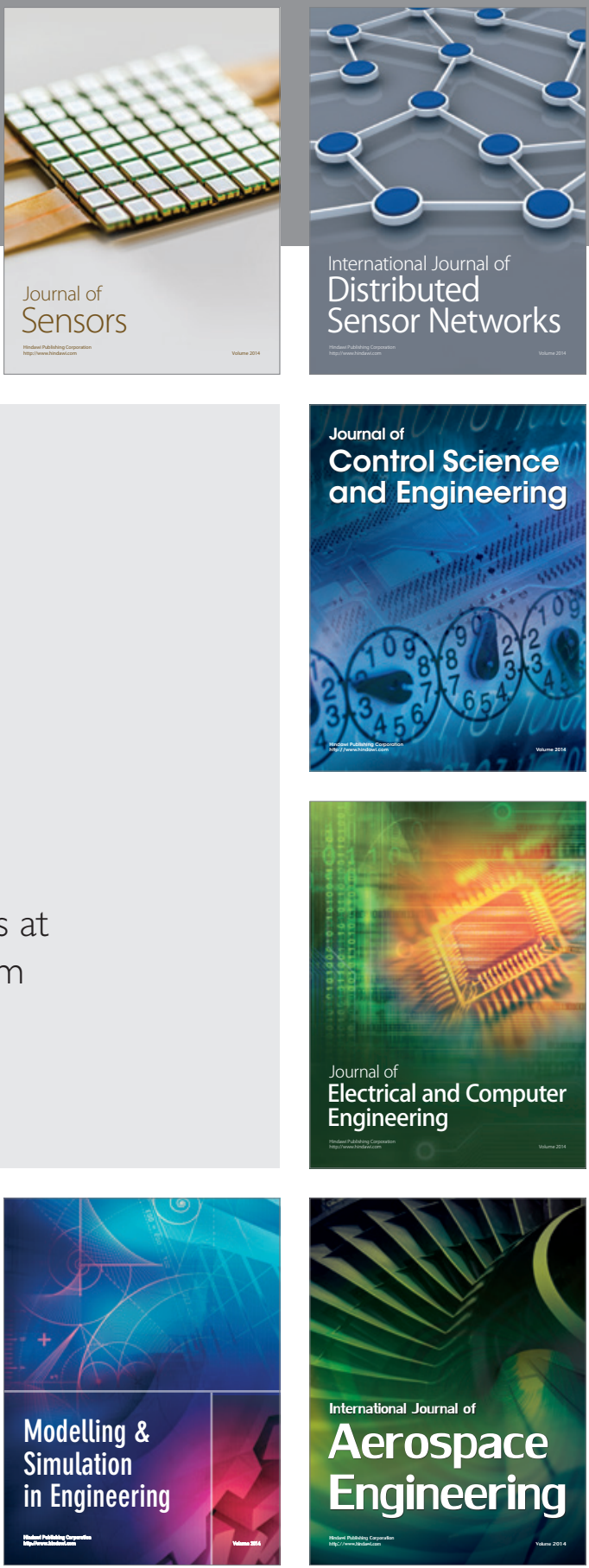

Journal of

Control Science

and Engineering
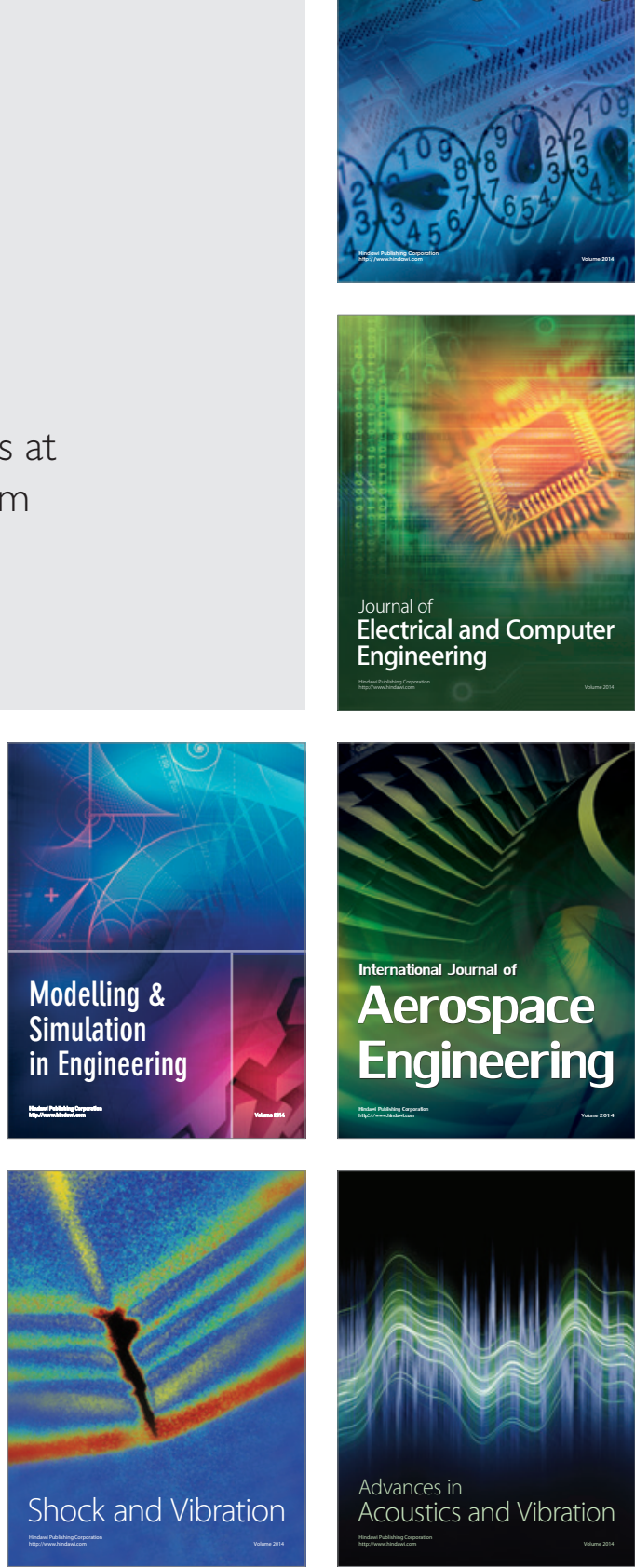